\title{
X-ray and optical observations of polars
}

\author{
Hauke Wörpel* \\ Leibniz-Institut für Astrophysik Potsdam \\ E-mail: hworpeleaip.de
}

\author{
Axel Schwope \\ Leibniz-Institut für Astrophysik Potsdam \\ E-mail: aschwopedaip.de
}

\begin{abstract}
The soft excess is a spectral feature shown by many polar cataclysmic variables, resembling a very luminous blackbody in soft X-rays. It is thought to arise from the accretion of discrete blobs of gas onto the WD, and was found in all polars discovered prior to the end of the ROSAT mission. All polars discovered since then seem to lack it, and the reason is still unknown.

Here we present an XMM-Newton study of four polars discovered optically in recent years. All four lack the soft excess, and we test the hypothesis that the sought-after spectral feature might be concealed in the very soft X-ray regime unobservable by XMM-Newton.
\end{abstract}

The Golden Age of Cataclysmic Variables and Related Objects IV 11-16 September, 2017

Palermo, Italy

* Speaker. 


\section{Introduction}

Polars are cataclysmic variables (CVs) in which the white dwarf primary possesses a magnetic field strong enough $(\sim 10-230 \mathrm{MG})$ to inhibit the formation of an accretion disc. The accreting material travels down the magnetic field lines of the primary onto relatively small regions near the magnetic pole, or both poles. A standing shock forms, causing the polar to emit copious X-rays in the form of a bremsstrahlung-like spectrum. This emission is isotropic, meaning that roughly one half strikes the surface of the white dwarf where it is then re-emitted as a blackbody-like component in extreme UV to very soft X-ray energies (e.g. [1]). Thus, the total flux of these two spectral components should be approximately equal.

Many polars, however, show very high flux in soft X-rays, far more than can be produced by the re-emission of bremsstrahlung. This soft excess was initially hard to explain but it is now generally accepted that it is caused by coherent blobs of gas surviving intact and penetrating deeply into the white dwarf atmosphere. Their energy then emerges in the form of a very bright and cool blackbody-like component [2,3], which can outshine the plasma emission by factors of a few tens. At times, the soft excess can actually be seen to be flare-like events superimposed on a plasma continuum (e.g. POS (GOLDEN 2017) 020).

The soft excess is a conspicuous identifying feature that led to many discoveries of polars with the Einstein, EUVE, EXOSAT, and ROSAT X-ray satellites. It was suspected to be present in all polars and, by the close of the ROSAT mission, over sixty soft excess polars had been discovered (e.g. [4]).

In the XMM-Newton era, however, no new soft excess polar has been discovered and it is not clear why. Polars previously known to have a soft excess continue to show it in XMM-Newton observations, but polars discovered after 2000 uniformly lack it. To address this mystery, we have performed XMM-Newton observations of four polar CVs initially identified as such by their optical spectra or light curves. That is, there is no bias in our sample regarding X-ray emission.

We here present the results of two papers [5, 6] detailing XMM-Newton, Swift, and optical observations of the four polars (V808 Aur, SDSS J032855.00+052254.2, SDSS J133309.20+143706.9, and 1RXS J173006.4+033813) discovered after the year 2000. The first of these is an eclipsing source, and is approximately one optical magnitude brighter that the other three. Our observations also serve to provide more information regarding the fluxes, magnetic field strengths, and energy distributions of polars generally.

\section{The targets}

The XMM-Newton observation of the eclipsing polar V808 Aurigae is the subject of [5]. We studied XMM-Newton and Swift observations of the other three sources, and a Swift observation of V808 Aur, in [6].

\subsection{V808 Aur}

V808 Aur is a deeply eclipsing polar discovered in the Catalina Sky Survey [7, 8, 9] with an orbital period of 117.18 minutes [10]. We were fortunate that the three X-ray observations caught V808 Aur in high, intermediate, and low accretion states. The brighter two observations, 
performed by XMM-Newton in 2012 and 2013, show typical accretion geometry: two accreting poles in approximately an offset dipole configuration with accretion primarily, but not exclusively, to one pole; see e.g. [11] for a review, though the actual magnetic field geometry is likely to be much more complex [12]. The X-ray light curves show the usual on/off behaviour as the primary pole rotates in and out of view.

The low accretion rate observation, taken by Swift in 2009, did not deliver enough photons for a spectral analysis but its temporal behaviour also seems to show, at $3.3 \sigma$ significance, accretion at both poles. We also found slight evidence that the orbital phase variation was present at UV wavelengths as well.

Optical spectroscopy performed at Calar Alto shows, via identification of cyclotron humps, magnetic field strengths of $36 \mathrm{MG}$ and $69 \mathrm{MG}$ at the primary and secondary poles respectively. Again, this is typical of the approximately dipolar magnetic field geometry common to polars.

None of the X-ray observations show any obvious soft excess, but did show a moderate soft component consistent with the re-radiation of bremsstrahlung. We therefore attempted to determine if a soft excess, in addition to the re-radiated plasma emission, could be present but unobserved in the region between soft X-rays and the XMM-Newton UV data point. Our reasoning was as follows: if there is such a blackbody, its low energy tail must not exceed the flux observed in UV, and the high energy tail should not cause the spectral fit in soft X-rays to deteriorate. Additional contraints involving the minimum possible blackbody temperature and maximum possible emitting area were also imposed, but turned out not to make very much difference.

We were thus able to rule out large portions of the blackbody temperature/normalisation parameter space. It is still possible, however, to conceal a very large soft excess for temperatures around $20 \mathrm{eV}$ and an emitting area of a few percent of the white dwarf surface (see Figure 14 of [5]).

The same approach was used to place restrictions on the soft excess for our other three targets as well.

It is worth noting that the moderate soft component we detected with XMM-Newton would have been detectable with ROSAT had V808 Aur been accreting in a high state at the time (which it was not). However, it would have been a faint and unremarkable source with an unexceptional hardness ratio, and would therefore likely have gone unidentified as a polar.

\subsection{SDSS J032855.00+052254.2}

This system is a polar with an orbital period of $121.97 \mathrm{~min}$, discovered in SDSS [13]. There are six X-ray observations of this source, two by XMM-Newton and four by Swift, taken within a period of six months in 2012.

The light curves show the on/off behaviour, similar to V808 Aur. They also show a very deep dip, caused by the accreting spot briefly passing behind the stream of accreting material. Both features can even be seen in the Swift data, which had to be pieced together from four short exposures.

X-ray spectroscopy of the bright phase shows a plasma continuum, but no soft component at all. Constraining the soft excess the same way we did for V808 Aur indicates that this component, if present, cannot be warmer than about $30 \mathrm{eV}$ and can arise from a region no larger than a few hundred kilometres in radius. 
We studied the SDSS spectra of this source and estimaed the magnetic field strength pole to be about $39 \mathrm{MG}$, slightly stronger than the $33 \mathrm{MG}$ inferred earlier by [13].

\subsection{SDSS J133309.20+143706.9}

A faint system discovered in SDSS, J1333 has an orbital period of 132 minutes [14]. We have one X-ray observation of this source, taken by XMM-Newton in 2012, but the source was in a low accretion state and little information could be obtained from the light curves. The phase-averaged $\mathrm{X}$-ray spectrum was consistent with a cool plasma, with a temperature of $4.3 \mathrm{keV}$, but the data were not of sufficient quality to look for a soft excess [6].

Our X-ray light curve showed no indication of variability over the orbital period. Long-term light curves from the Catalina Sky Survey show that this source spends almost all of its time accreting at a low level. It is therefore not a good candidate for seeking the soft excess.

\subsection{RXS J173006.4+033813}

J1730 was detected as a moderately bright, but otherwise unremarkable, point source in the ROSAT All-Sky Survey [15] but not recognized as a polar until later. Optical photometry gives a period of 120.21 minutes [16]. We obtained an XMM-Newton observation of this source in February 2012. There are also archival Swift observations but, since these were presented already in [16] we have not re-analysed them.

Our X-ray data again show the on/off light curves of a primary accreting pole rotating in and out of view. There is also an accretion dip, though not as prominent as for SDSS J0328. The spectrum is, as expected, a plasma with temperature of around $15 \mathrm{keV}$ as well as a modest soft component of $60 \mathrm{eV}$ consistent with re-emitted plasma emission.

As we found for V808 Aur and SDSS J0328, a very significant blobby accretion blackbody could be concealed in the unobservable part of the spectrum, again with a temperature around 20$30 \mathrm{eV}$ and an emitting area of around $1 \%$ of the surface area of the white dwarf. This scenario does not seem implausible, thus a significant soft excess in this source cannot be ruled out.

For this source we also obtained an optical spectrum using the CAFOS spectrograph on the $2.2 \mathrm{~m}$ telescope at Calar Alto. In this observation we were unable to detect individual cyclotron harmonics. If the magnetic field strength were higher than about $40 \mathrm{MG}$ we would most likely have been able to distinguish these features, suggesting that the field strength is significantly lower. Indeed, the cyclotron continuum looks similar to those of MR Ser and V834 Cen [17, 18], which have field strengths of around $25 \mathrm{MG}$ so we conclude that J1730 also has a field strength of around this value.

\section{Conclusion}

We have studied four polars not previously observed by XMM-Newton, primarily to search for the soft excess ubiquitous in polars discovered prior to 2000 but missing in new polars thereafter. We found no obvious sign of it. There is still a possibility that, if the soft blobby emission has a cool temperature, it could be very luminous and nevertheless not be detectable by XMM-Newton.

We devised a method of testing this hypothesis based on the reasoning that, if such a blackbody is present, its red and blue tails must not conflict with the XMM-Newton UV and X-ray points that 
we can measure. A blackbody with a temperature of $20-30 \mathrm{eV}$ and an emitting area of around $1 \%$ of the WD area can outshine the plasma emission by a factor of a few tens and still escape detection. We therefore cannot rule out that the soft excess is present but undetectable. It is difficult to explain though why all post-ROSAT polars should hide the soft excess in this manner when none of the previously known ones did.

The strange dichotomy between polars discovered in the ROSAT era, which all show a prominent soft excess, and those discovered later, which all seem not to have it, is only partly explicable. Sources such as V808 Aur and 1RXSJ1730 exhibit a modest soft component, enough to be detectable by ROSAT, but appear as unremarkable point sources. It is not that they were undetected, but that they were hard to identify.

Explaining why no new soft excess polars have been discovered since 2000 is harder. Though XMM-Newton is primarily a pointed instrument, it is capable of discovering magnetic CVs serendipitously (e.g. 2XMMp J131223.4+173659 and 2XMMi J225036.9+573154, [18] and [19] respectively). Furthermore, the XMM-Newton slew survey [20] is of comparable sensitivity to the RASS, though of course with somewhat lower sky coverage. It is hard to believe that the soft excess polars could have escaped discovery by chance. The next major X-ray survey mission, the eROSITA mission due to launch in 2019 [21], has excellent prospects of uncovering new soft excess polars, and to place more stringent limits on how common they are if it does not find any.

Aside from studying the soft excess, our work also adds information to our knowledge of the light curves, X-ray spectra and luminosities, and energy distributions of polars generally. Where optical spectra are available, we have also measured the magnetic field strengths and found them to be fairly typical for polars with strengths of a few tens of megagauss.

\section{References}

[1] M. Cropper, The Polars, Space Sci. Rev. 54195 (1990)

[2] J. Kuijpers \& J. Pringle, Comments on radial white dwarf accretion, A\&A 114, 4 (1982)

[3] G. Ramsay \& M. Cropper, The Energy Balance of Polars revisited, MNRAS 347, 497 (2004) [astro-ph/0309527]

[4] H. C. Thomas, K. Beuermann, V. Burwitz, K. Reinsch, \& A. D. Schwope RX J1313.2-3259, $a$ long-period Polar discovered with ROSAT, A\&A 353, 646 (2000) [astro-ph / 9911200 ]

[5] H. Worpel \& A. D. Schwope, XMM-Newton and optical observations of the eclipsing polar CSS081231:071126+440405, A\&A 583, 130 (2015) [astro-ph/1507.02411 ]

[6] H. Worpel, A. D. Schwope, T. Granzer, K. Reinsch, R. Schwarz \& I. Traulsen, X-ray and optical observations of four polars, A\&A 592114 (2016) [astro-ph/1605.00927]

[7] A. J. Drake, S. G. Djorgovski, A. Mahabal, et al First Results from the Catalina Real-Time Transient Survey, ApJ 696, 870 (2009) [astro-ph/ $0809.1394 \mathrm{v} 2$ ]

[8] D. V. Denisenko \& S. Korotkiy OT_J071126.0+440405 eclipses and period measurement vsnet-alerts 10870 (2009)

[9] M. Templeton, A. Oksansn, D. Boyd, R. Pickard \& H. Maehara, Variable Star in Auriga, Central Bureau for Astronomical Telegrams 1652 (2009) 
[10] A. D. Schwope, F. Mackebrandt, B. D. Thinius, C. Littlefield, P. Garnavich, A. Oksanen, A. \& T. Granzer, Multi-epoch time-resolved photometry of the eclipsing polar CSS081231:071126+440405, AN 336, 115 (2015) [astro-ph/1501.05217]

[11] D. T. Wickramasinghe \& L. Ferrario, Magnetism in Isolated and Binary White Dwarfs, PASP 112, 873 (2000)

[12] K. Beuermann, F. Euchner, K. Reinsch, S. Jordan, \& B. Gänsicke, Zeeman tomography of magnetic white dwarfs. IV. The complex field structure of the polars EF Eridani, BL Hydri and CP Tucanae, $A \& A$ 463, 647 (2007) [astro-ph/ 0610804 ]

[13] P. Szkody, A. Henden, L. Manikko, et al., Cataclysmic Variables from Sloan Digital Sky Survey. VI. The Sixth Year (2005), AJ 134, 185 (2007)

[14] P. Szkody, S. F. Anderson, M. Hayden, et al., Cataclysmic Variables from SDSS. VII. The Seventh Year (2006), AJ 137, 4011 (2009) [astro-ph/0 901 . 3177]

[15] W. Voges, B. Achenbach, T. Boller et al., The ROSAT all-sky survey bright source catalogue, A\&A 349, 389 (1999) [astro-ph/9909315]

[16] V. B. Bhalerao, M. H. van Kerkwijk, F. A. Harrison, M. K. Kasliwal, S. R. Kulkarni, \& V. R. Rana, The Polar Cataclysmic Variable 1RXS J173006.4+033813, ApJ 721, 412 (2010) [astro-ph/1008.2002]

[17] A. D. Schwope, K. Beuermann, S. Jordan, \& H.-C. Thomas, Cyclotron and Zeeman spectroscopy of MR Serpentis in low and high states of accretion, A\&A 278, 784 (1993)

[18] A. D. Schwope \& K. Beuermann, Cyclotron and Zeeman spectroscopy of V834 Centauri, A\&A 238, 173 (1990)

[18] J. Vogel, K. Byckling, A. D. Schwope, J. P. Osborne; R. Schwarz, \& M. G. Watson, The serendipituous discovery of a short-period eclipsing polar in $2 X M M p, A \& A 485,787$ (2008) [astro-ph/0804.3946]

[19] G. Ramsay, S. Rosen, P. Hakala, \& T. Barclay, 2XMMiJ225036.9+573154 - a new eclipsing AMHer binary discovered using XMM-Newton, MNRAS 395, 416 (2009) [astro-ph / 0901 . 3095]

[20] R. D. Saxton, A. M. Read, P. Esquej, M. J. Freyberg, B. Altieri, \& D. Bermejo. The first XMM-Newton slew survey catalogue: XMMSL1, A\&A 480, 611 (2008) [astro-ph/0801.3732]

[21] A. Merloni, P. Predehl, W. Becker, et al., eROSITA Science Book: Mapping the Structure of the Energetic Universe (2012) [astro-ph/1209.3114] 